\title{
A Heart Sound Segmentation Method Based on Cyclostationary Envelope
}

\author{
Ting $\mathrm{Li}^{1,2}$, Tianshuang Qiu${ }^{1}$, Hong Tang ${ }^{1}$ \\ ${ }^{1}$ Faculty of Electronic and Electrical Engineering, Dalian University of Technology, Dalian, \\ 116024, China \\ ${ }^{2}$ College of Information and Communication Engineering, Dalian Nationalities University, \\ Dalian, 116600, China \\ e-mail: liting@dlnu.edu.cn
}

\begin{abstract}
Segmentation is generally an important prior process to automatic analysis of heart sounds. The proposed algorithm achieved this in separated cycles. First, the cyclostationary envelopes of heart sound signals are calculated. Second, a threshold is chosen to separate S1 and S2. The segmentation is automatic, and robust to noise. It can identify S1 and S2 correctly even under the circumstance of noise and interference. No reference signal is needed for this segmentation. It was tested by the heart sounds of 20 subjects including 15 normal and 5 abnormal in various clinical cases (715 cycles in total). The statistics showed over 96 percent correctness for segmentation.
\end{abstract}

Keywords-Heart sound signal; Segmentation;
Cyclostationary Envelope

\section{INTRODUCTION}

Phonocardiography is a noninvasive, easy, and efficient method to evaluate heart functions. Heart auscultation is widely used in medical check-up for adults and children due to its easy operation and economy. In auscultation, physicians or cardiologists try to separate heart sound signal into four phases, i.e., first heart sounds (S1), systolic phase, second heart sounds (S2), diastolic phase, and then analyze the features. However, heart sounds are transient and fast varying. Heart sounds analysis by human ears highly depends on the skills and experience of the listeners. Therefore, it is desirable to analyze the recording of heart sounds in a computerized way. Segmentation is generally a very important pre-process for automatic analysis of heart sounds, i.e., to identify the timings and the time durations of S1, the systolic phase (or systolic murmur), S2 and the diastolic phase (or diastolic murmur) in this sequence in time.

The previous studies of segmentation have been reported in literature. In the past, ECG signals were always used as reference signals in the heart sound segmentation methods [1]. Liang et al. identified S1 and S2 using the Shannon envelope [2]. Wang et al. improved this algorithm by changing two-order Shannon envelope to three-order [3]. Wang Xinpei et al. proposed a segmentation method based on Shannon entropy [4]. These methods mentioned above have a common problem that the segmentation results are not good when noise and interference occur. This disadvantage restricts the applications of these methods in practice because noise and interference are inevitable in the process of heart sound signals collecting.
The objective of this paper is to develop a robust heart sound signal segmentation method based on the cyclostationary envelope. The heart sound signals are cyclostationary while noise and interference are not. So the cyclostationary envelope can get rid of the effects of noise and interference. That is to say, the heart sound signals can be correctly segmented even if they are noisy. The paper is organized as follows. Representation of cyclostationary envelope is given in section 2. Segmentation method is proposed in section 3. Computer simulations for heart sounds of different clinical cases and robustness of the algorithm to noise are evaluated in section 4 . Conclusions are given in section 5 .

\section{REPRESENTATION OF CYCLOSTATIONARY ENVELOPE}

Heart sound signals which are quarsi-periodical and nonstationary have obvious cyclostationary characteristics [5-7]. The envelope of heart sound signals can be indicated by a linear sum of cycle frequency, in which the linear coefficients can be estimated by the cyclostationary spectrum [8].

Here we study the cyclostationary characteristics of heart sound signals through two-order cyclic spectral coherence function (CSCF), which is defined as

$$
\rho_{x}^{\alpha}(f)=\frac{S_{x}^{\alpha}(f)}{\left[S_{x}^{0}(f-\alpha / 2) S_{x}^{0}(f+\alpha / 2)\right]^{1 / 2}}
$$

where $\rho_{x}^{\alpha}(f)$ which is the function of $\alpha$ and $f$ shows the cyclostationarity strength of a signal at cycle frequency $\alpha$ and frequency $f$. $S_{x}^{\alpha}(f)$ is the cyclic spectrum of $x(t)$ at the cycle frequency $\alpha . S_{x}^{\alpha}(f)$ and $S_{x}^{0}(f-\alpha / 2)$ are written as

$$
\begin{gathered}
S_{x}^{\alpha}(f)=\int_{-\infty}^{\infty} R_{x}^{\alpha}(\tau) e^{-j 2 \pi f \tau} d \tau \\
S_{x}^{0}(f-\alpha / 2)=\int_{-\infty}^{\infty} R_{x}^{0}(\tau) e^{-j 2 \pi(f-\alpha / 2) \tau} d \tau \\
R_{x}^{\alpha}(\tau)=<x(t+\tau / 2) x^{*}(t+\tau / 2) e^{-j 2 \pi \alpha}>_{t}
\end{gathered}
$$

where $R_{x}^{\alpha}(\tau)$ is called as the cyclic correlation function, and the operator $\langle\bullet\rangle_{t}$ denotes time average. In order to describe the energy distribution of heart sound signals more clearly in the cycle frequency domain, $\rho_{x}^{\alpha}(f)$ is transformed as the function of $\alpha$ only, i.e., 


$$
G_{x}(\alpha)=\int_{f_{a}}^{f_{b}} \rho_{x}^{\alpha}(f) d f
$$

where $f_{a}$ and $f_{b}$ are the upper frequency limit and lower frequency limit of stronger heart sound signal components, respectively; $G_{x}(\alpha)$ which is cyclic coefficient is the function of $\alpha$. This coefficient denotes the cyclostationarity strength of this signal at cycle frequency $\alpha$. Then in the cycle frequency domain, the cyclostationary envelope of heart sound signal $x(t)$ is defined as

$$
c(t)=\sum_{\alpha=0}^{\infty} G_{x}(\alpha) e^{j 2 \pi \alpha}
$$

That is to say, the cyclostationary envelope of heart sound signal $x(t)$ is the linear combination of cyclic coefficient $G_{x}(\alpha)$ and cycle frequency $\alpha . G_{x}(\alpha)$ is decreased with the increasing of $\alpha$. When $G_{x}(\alpha)$ is so small that $G_{x}(\alpha)$ has little contribution to the envelope of heart sound signals, these $G_{x}(\alpha)$ can be omitted. Therefore, in the calculation of the cyclostationary envelope, only several $\alpha(n)(n=1,2, \ldots, N)$ with more energy need to be considered. Equation (6) is rewritten as

$$
c_{N}(t)=\sum_{n=1}^{N} G_{x}(\alpha(n)) e^{j 2 \pi \alpha(n) t}
$$

where $c_{N}(t)$ indicates the envelope combined by $N$ bigger $G_{x}(\alpha(n))$.

\section{SEGMENTATION OF THE CYCLOSTATIONARY ENVELOPE}

We can get the cyclostationary envelopes of heart sound signals according to the process described in section 2. The summits of the envelopes basically imply the positions of S1 and S2. In order to determine the accurate boundaries of S1 and S2, a threshold is set to search the beginnings and ends of S1 and S2. Then time gates of S1 and S2 are constructed, and the segmentation of cyclostationary envelopes is realized finally. The steps are as follows.

i. A threshold is chosen according to the amplitude of the envelope, and the amplitudes larger than the threshold are considered as S1 and S2.

ii. Construct the time gates of S1 and S2. Set the amplitudes larger than the threshold 1 and others 0 , which can make the results of segmentation more obviously.

iii. Correspond the beginning and end points to the former heart sound signals and mark S1 and S2 on them.

Because of the complexity of heart sound signals, the results of segmentation may not be correct sometimes if only a threshold is used. Especially in the situation of heart sound splits, the cyclostationary envelope will be split too, which makes redundant time gates occur. Getting rid of redundant time gates is needed before identifying S1 and S2.

i. Calculate the intervals between all neighboring time gates, and set a lower limit L1 according to the mean and variance of the intervals. ii. Compare the intervals to the lower limit L1. If the interval is smaller than L1 and smaller than 50 ms (the maximum interval of normal heart sound split), these two time gates are considered as the heart sound split and will be combined.

iii. If the interval is smaller than $\mathrm{L} 1$ and larger than $50 \mathrm{~ms}$, the latter time gate is thought to be murmurs and will be wiped off.

iv. Identify heart sound period on the basis of physiological principles. Because the maximum interval of two neighboring time gates are diastoles (from the end of S2 to the beginning of S1) [2], the only thing to do is to find the biggest interval and mark the former time gate S2 and the latter one S1. Other time gates are signed according to this.

\section{SIMULATION RESULTS AND DISCUSSIONS}

The heart sound data are downloaded from Internet or collected in the authors' lab. All heart sound data are preprocessed as follows. First, heart sound signals are filtered by linear low-pass filters whose stop frequency is $800 \mathrm{~Hz}$. Second, heart sound signals are down sampled to 2 $\mathrm{kHz}$. Third, heart sound signals are normalized.

A segmentation result is shown in Fig. 1. Fig. 1 (a) is a normal heart sound signal with noise free, Fig. 1 (b) is the cyclostationary envelope of (a), Fig. 1 (c) is the corresponding time gates, and Fig. 1 (d) is the segmentation results.

Fig. 2 is another example. Fig. 2 (a) is a normal heart sound signal with noise, and the SNR is $1 \mathrm{~dB}$; Fig. 2 (b) is the cyclostationary envelope of (a), Fig. 2 (c) is the corresponding time gates, and Fig. 2 (d) is the segmentation results. From the result, we can see that the cyclostationary envelope is not affected by noise, that is to say, the algorithm proposed here can identify S1 and S2 correctly even under the circumstance of noise, whose performance is much better than those methods before.

The proposed segmentation was tested by various clinical heart sound signals, including normal heart sound signal, mitral regurgitation, aortic stenosis, aortic regurgitation, pulmonary stenosis, and S1 split, respectively. These are totally 715 cycles from 20 subjects, in which 15 are normal and 5 are abnormal. S1s and S2s were firstly segmented in hand and then we compared the results with those of the proposed algorithm. The situation of noise and noise free are both tested. Correct percentage of identification of S1and S2 was given in Table 1 . The results indicated over 96 percent correctness in the identification of S1 and S2. Large murmurs that overlap S1 and S2 will make the correct identification impossible. However, because the murmur is large, it can not be neglected by auscultation.

\section{CONCLUSION}

The automatic segmentation algorithm is found to effective to segment phonocardiogram signals. Even under noise, the proposed algorithm can achieve over 96 percent success in 20 recordings, which include 715 cycles of heart sounds. This is a good basis for further analysis of the heart sound signals. With this segmentation, we can extract the features of each segment, such as the root mean square, the 
peak intensity, the peak location the duration, the splitted interval of S1 and S2, etc. We can also do other processed to each segment for the classification.

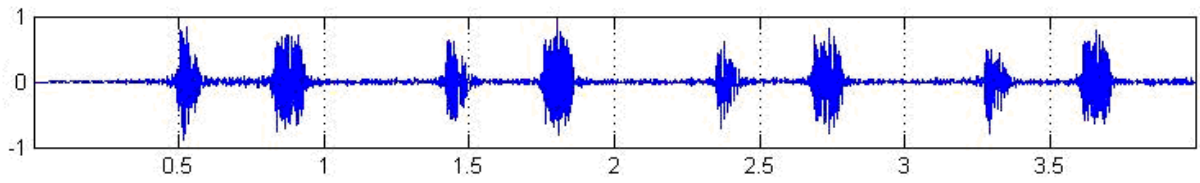

(a) time (s)

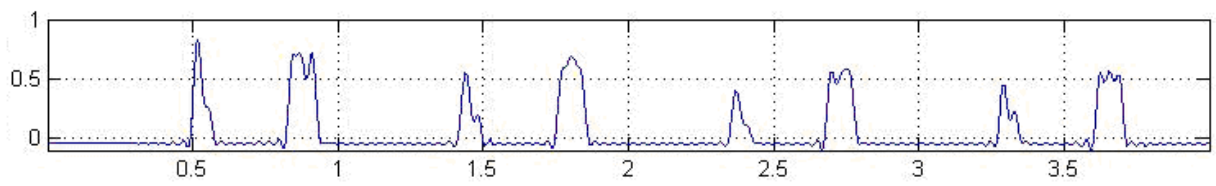

(b) time (s)

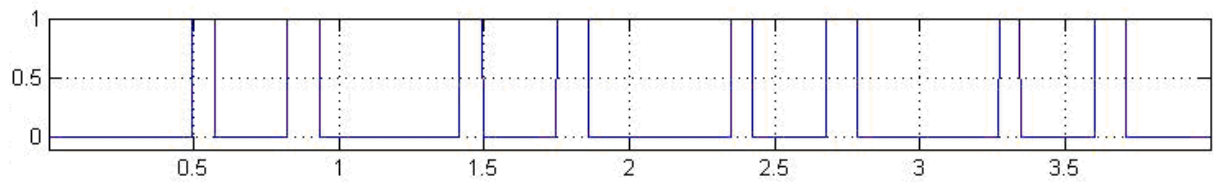

(c) time (s)

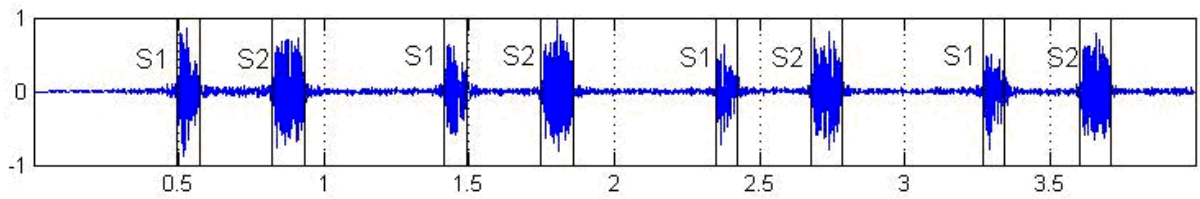

(d) time (s)

Figure 1. Segmentation results of a normal heart sound signal without noise. (a) A normal heart sound signal with noise free. (b) The cyclostationary envelope of (a). (c) The corresponding time gates. (d) The segmentation results.

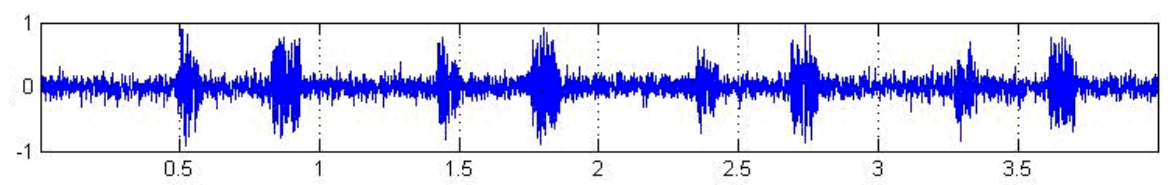

(a) time (s)

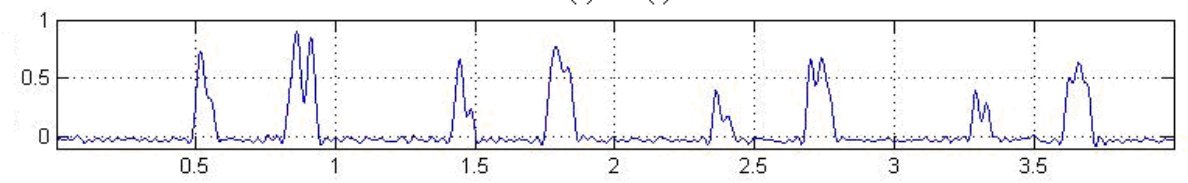

(b) time (s)

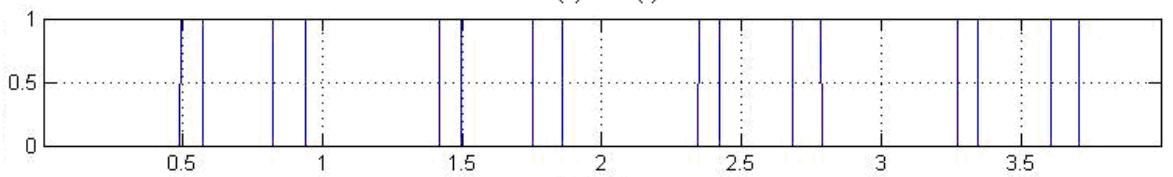

(c) time (s)

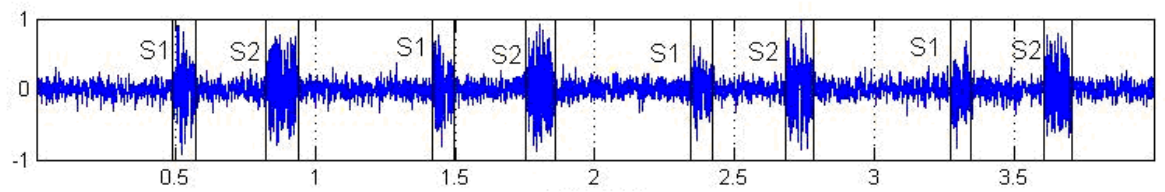

(d) time (s)

Figure 2. Segmentation results of a normal heart sound signal with noise. (a) A normal heart sound signal with noise, SNR is $1 \mathrm{~dB}$. (b) The cyclostationary envelope of (a). (c) The corresponding time gates. (d) The segmentation results. 
TABLE I. STATISTICS OF SEGMENTATION CORRECTNESS

\begin{tabular}{|c|c|c|}
\hline & Noise free & Noisy \\
\hline Number of cycles & 715 & 715 \\
\hline Correct & 702 & 691 \\
\hline Percentage & $98.2 \%$ & $96.6 \%$ \\
\hline
\end{tabular}

\section{REFERENCES}

[1] Groch MW, Domnanovich JR, et al. A new heart sounds gating devices for medical imaging. IEEE Trans. on Biomedical Engineering, 1992, 39: 307-310.

[2] Liang H, Lukkarinen S, et al. Heart sound segmentation algorithm based on heart sound envelope. Computers in Cardiology, 1997, 24: 105-108.

[3] Wang XP, Li YY, et al. Detection of the first and second heart sound using heart sound energy. 2nd International Conference on Biomedical Engineering and Informatics, 2009: 1-4.

[4] Wang Xinpei, et al. Heart sound segmentation algorithm based on high-order Shannon entropy. Journal of Jilin Unversiity (Engineering and Technology Edition), 2010, 40(5): 1433-1437.
[5] T. Li, H. Tang, T. Qiu. Best subsequence selection of heart sound recording based on degree of sound periodicity. IEE Electronics Letters, 2011, 47(15): 841-843.

[6] Hong Tang, Ting Li, Tianshuang Qiu. Noise and disturbance reduction for heart sounds in the cycle frequency domain based on non-linear time scaling. IEEE Transactions on biomedical engineering, 2010, 57(2): 325-333.

[7] Hong Tang, Ting Li, Tianshuang Qiu, et al. Separation of heart sound signal from noise in joint cycle frequency-time-frequency domains based on fuzzy detection, IEEE Transactions on Biomedical Engineering, 2010, 57(10): 2438-3447.

[8] Tang Hong, Li Ting, et al. Cardiac sound envelope synthesis in cycle frequency domain. Chinese Journal of Biomedical Engineering, 2009, 28(5):662-667. 